\title{
Sistemas electrónicos de administración de nicotina: ¿una amenaza para el neurodesarrollo de los adolescentes?
}

\section{Electronic Nicotine Delivery Systems: A Threat to the Neurodevelopment of Adolescents?}

Felipe Botero-RodrígueZ ${ }^{\mathrm{a}}$

Médico, Facultad de Medicina, Pontificia Universidad Javeriana, Bogotá, Colombia. Departamento de Epidemiología Clínica y Bioestadística, Facultad de Medicina, Pontificia Universidad Javeriana, Colombia

Arturo Marroquín Rivera Médico, Facultad de Medicina, Pontificia Universidad Javeriana, Bogotá, Colombia. Departamento de

Epidemiología Clínica y Bioestadística, Facultad de Medicina, Pontificia Universidad Javeriana. Joven investigador de Colciencias, Colombia

\section{Alejandra Leal}

Residente de Psiquiatría, Pontificia Universidad Javeriana, Bogotá, Colombia

Camilo Cabarique Méndez

Departamento de Epidemiología Clínica y

Bioestadística, Facultad de Medicina, Pontificia Universidad Javeriana, Bogotá, Colombia. Médico

psiquiatra, Facultad de Medicina, Pontificia

Universidad Javeriana, Bogotá, Colombia

Carlos Gómez-Restrepo

Departamento de Epidemiología Clínica y

Bioestadística, Facultad de Medicina, Pontificia

Universidad Javeriana. Médico psiquiatra, Departamento de Psiquiatría y Salud Mental, Facultad de Medicina, Pontificia Universidad Javeriana, Hospital Universitario San Ignacio, Bogotá, Colombia

a Correspondencia: fboterorodriguez@hotmail.com

Cómo citar: Botero-Rodríguez F, Marroquín Rivera A, Leal A, Cabarique Méndez C, Gómez-Restrepo C. Sistemas electrónicos de administración de nicotina: ¿una amenaza para el neurodesarrollo de los adolescentes? Univ. Med. 2019;60(4). https:// doi.org/10.11144/Javeriana.umed60-4.sean

\section{RESUMEN}

Introducción: Los cigarrillos electrónicos, introducidos en el mercado en el 2003 y con gran acogida comercial desde entonces, se han convertido en un dispositivo que puede igualar o superar el riesgo del cigarrillo de combustión respecto a múltiples desenlaces nocivos para la salud. A pesar de ello, estos dispositivos se perciben como menos lesivos o adictivos, y son más aceptados socialmente. Esta nueva práctica ha sido especialmente acogida por la población adolescente, época de la vida en la que existe una mayor vulnerabilidad debido a su proceso de neurodesarrollo. Objetivo: Realizar una revisión temática de los cigarrillos electrónicos, sus políticas de regulación, sus patrones de consumo y sus asociaciones con la salud mental. Métodos: Se realizó una búsqueda de estudios secundarios, 
en PubMed y SciELO, utilizando términos MeSH (o DeCS) y libres. Posteriormente, se revisaron los artículos obtenidos y se filtraron teniendo en cuenta los temas que se iban a evaluar. Después de realizar el proceso de tamización, se obtuvieron 54 artículos. Discusión: Se describe la influencia de la nicotina en el desarrollo cerebral del adolescente, así como la tendencia del consumo de cigarrillos electrónicos y las medidas regulatorias que se han implementado. El riesgo elevado para la salud mental de los adolescentes que representan los cigarrillos electrónicos, sumado a una pobre regulación en Colombia, genera la necesidad de fortalecer los sistemas de prevención e intervención para el consumo de nicotina, así como políticas comerciales más rigurosas frente a su venta en diferentes modalidades.

Palabras clave

sistemas electrónicos de liberación de nicotina; cigarrillo electrónico; adolescente; salud pública; salud mental.

\begin{abstract}
Introduction: Electronic cigarettes were introduced in the market in 2003. Since then they have become a device broadly used worldwide with an important commercial activity. Unfortunately, they have an equivalent or even greater risk for multiple harmful health outcomes when compared with conventional cigarettes. Nevertheless, these devices are perceived less harmful or addictive, and are more socially accepted. Additionally, most users belong to the teenage population, which is characterized by greater vulnerability due to the ongoing neurodevelopment process. Objective: Conduct a thematic review of the electronic cigarettes, their regulatory policies, consumption patterns and described associations with mental health. Methodology: A search for secondary studies was conducted in PubMed and SciELO databases using $\mathrm{MeSH}$, DeCS and free terms. Review results were performed according to the aimed topics for evaluation. Finally, 54 articles were obtained after the screening process. Discussion: The influence of nicotine on adolescent brain development is well described, just as their consumption trend and the regulatory measures that have been implemented. The high risk for mental health in adolescents that represent electronic cigarettes, added to poor regulations in our country, generates the need to strengthen prevention and intervention systems for nicotine consumption, as well as stringent commercial policies against its sale modalities.
\end{abstract}

Keywords

electronic nicotine delivery systems; electronic cigarette; adolescent; public health; mental health.

\section{Introducción}

Los cigarrillos electrónicos (CE) hacen parte de los sistemas electrónicos de administración de nicotina (SEAN). Estos se introdujeron en el mercado en 2003, y cuatro años después ya se había registrado y patentado su diseño comercial en múltiples países. Son dispositivos que producen aerosol a partir del calentamiento de líquidos (líquido E) que contienen solventes (glicerina vegetal, propilenglicol o una mezcla de estos), uno o más saborizantes y nicotina, aunque esta última es opcional $(1,2,3,4,5)$. La evaporación del líquido $\mathrm{E}$ en la porción del dispositivo que es calentado es seguida rápidamente por un enfriamiento. Este proceso genera el aerosol que será inhalado (o vapeado como suele referirse a la inhalación al usar CE) por el usuario. Cada dispositivo se conforma a partir de una batería que calienta el líquido, un reservorio donde se contiene este último, un atomizador que se encarga de generar calor y una cámara de vaporización $(1,2,3,6,7)$.

En condiciones típicas, la exposición a las potenciales toxinas de sus componentes es menor, comparado con los cigarrillos de combustión; sin embargo, el uso inadecuado de sus componentes puede igualar o aumentar el riesgo. Adicionalmente, la inclusión de una variedad de sabores en los CE incita a más personas a consumir estos productos, sobre todo a quienes se encuentran en etapa contemplativa para el cese de consumo de tabaco. Por esta variedad de sabores y su uso en algunas terapias antitabaco, los CE son percibidos como menos dañinos, menos adictivos y más aceptados socialmente, por lo que generan una falsa sensación de seguridad $(8,9,10)$. No obstante, la evidencia de los mecanismos fisiopatológicos, en cuanto a sintomatología, desenlaces y efectos de los $\mathrm{CE}$, sugieren un efecto nocivo en los humanos (8). Además, análisis químicos de los dispositivos comercialmente disponibles han encontrado nicotina en productos etiquetados como libres de esta sustancia, así como la presencia de sustancias tóxicas como dietilenglicol y formaldehído $(11,12,13)$. Esta exposición a la nicotina lleva a adicción y alterar el desarrollo cerebral, al producir alteraciones en el aprendizaje, la memoria y la atención (14).

Un aspecto preocupante de estos productos es su creciente popularidad comercial. 
Especialmente el JUUL, un tipo particular de SEAN que se ha vuelto muy popular en los últimos tres años, pues para el 2018 dominaba el mercado con casi el $70 \%$ de las ventas en Estados Unidos. Su popularidad se debe principalmente a su pequeño tamaño y, por ende, a la facilidad de sostenerlo. También, dado que se carga a través de un puerto para USB y usa JUULpods, con disponibilidad de múltiples sabores y concentración de nicotina, que puede ser equivalente a un paquete de cigarrillos de combustión $(7,15)$.

El objetivo de este artículo es revisar la tendencia del consumo de CE, su asociación con condiciones de salud mental y las medidas regulatorias que se han implementado para el control de los SEAN.

\section{Metodología}

Se realizó una búsqueda electrónica y sistemática en Medline (por medio del motor de búsqueda Pubmed) y en SciELO durante junio del 2019. Se usaron términos MeSH y términos libres que incluían e cigarrette, electronic cigarrette, mental health, adolescent, public health, regulations y addiction. Adicionalmente, se especificó que no se incluyeran estudios referentes a problemas pulmonares con el conector booleano NOT y los términos neum* y pulm*. También se utilizaron los términos DeCS correspondientes.

Se incluyeron trabajos secundarios que hablaran sobre los patrones de consumo, regulaciones legales y comerciales, salud pública, frecuencia de uso y afecciones de salud mental. Se descartaron aquellos artículos que trataran específicamente de toxicidad pulmonar o cardiaca, riesgo materno en el embarazo o riesgo de explosión y lesiones en piel. Si no existían revisiones sobre uno de los temas enfatizados, se seleccionaban artículos primarios referenciados en los trabajos secundarios y se expandía la búsqueda en Google Académico. No se excluyeron artículos por idioma, ni se limitó la búsqueda por fecha de publicación. Adicionalmente, cabe aclarar que se descartaron artículos de opinión y resúmenes publicados o expuestos en congresos y libros.

Una vez se contó con todas las fuentes bibliográficas, se filtraron teniendo en cuenta título y resumen. Cuatro de los autores realizaron conjuntamente la filtración por título y resumen. Posteriormente, se leyeron la totalidad de los artículos seleccionados y se descartaron los que no contuviesen la información necesaria.

\section{Resultados}

En la figura 1 se expone el proceso de selección, donde se obtuvieron 233 artículos, de los cuales se descartaron 161 por título y resumen. Posteriormente, se descartaron 23 al leer el texto completo, por el tipo de estudio o el contenido no concordante con los objetivos de la revisión. Adicionalmente, en busca de regulaciones locales y profundización de los temas, por medio de las referencias y Google Académico, se adicionaron 8 trabajos. A partir de estos estudios se estructuraron los temas que componen la revisión.

\section{Figura 1}

Proceso de obtención de artículos

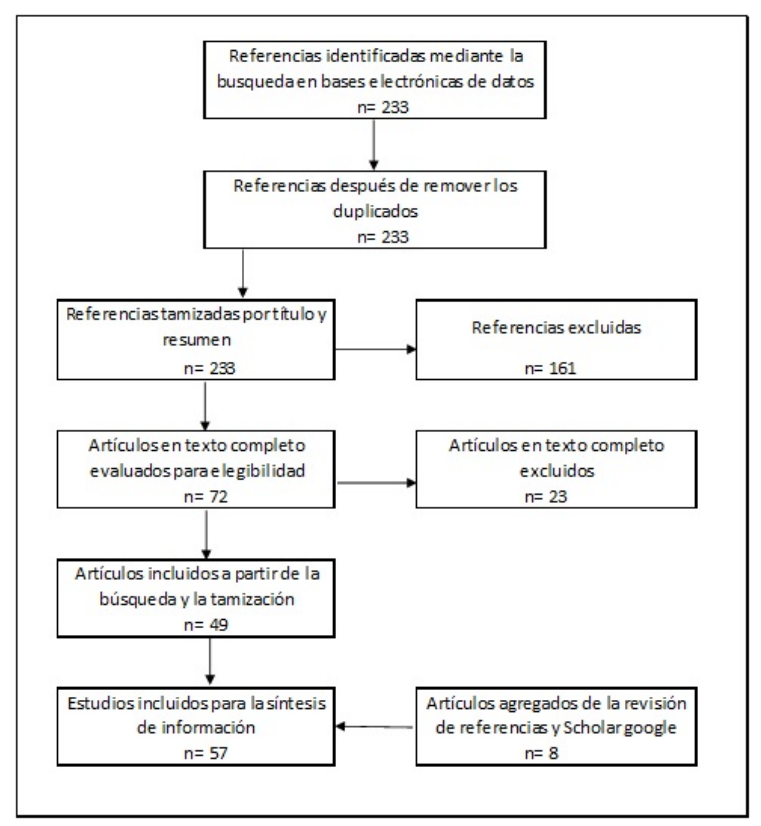


Fisiología de la concentración sanguínea del cigarrillo de combustión en contraposición al cigarrillo electrónico

Cuando se utilizan CE, la concentración máxima de nicotina en la sangre se suele alcanzar en un periodo más extenso. En la primera generación de $\mathrm{CE}$, las concentraciones máximas eran más bajas que los cigarrillos de combustión; sin embargo, a medida que se han desarrollado nuevas generaciones, ha aumentado la concentración máxima. Esta puede, incluso, superar las concentraciones alcanzadas con el consumo de cigarrillos de combustión. Las concentraciones máximas son de $43,6 \mathrm{ng} / \mathrm{ml}$ y $18 \mathrm{ng} / \mathrm{ml}$ para los CE y los tradicionales, respectivamente. $\mathrm{Al}$ aumentar la concentración de la nicotina en el líquido $\mathrm{E}$, aumenta la concentración máxima en la sangre bajo una relación linear $(16,17)$. Y aunque estos se suelen tener menor cantidad de sustancias toxicas, persiste un número importante de carcinógenos susceptibles de ser inhalados (18). Esto último es ambiguo, y no hay consenso acerca de los efectos de los CE en los pacientes con cáncer (19).

La cantidad de inhalaciones también es proporcional a la concentración de nicotina en la sangre, lo que produce un mayor umbral de satisfacción, que cada vez es más difícil de superar. Además, aquellos que fumaban cigarrillo previamente tienen un umbral de satisfacción mayor (17). El aumento en este, asociado a que la población adolescente es más susceptible a su uso, hace fundamental evaluar los efectos deletéreos del CE $(5,20)$. Además, se conoce que la adolescencia es una época de la vida relacionada con un mayor riesgo de consumo de sustancias $(21,22)$. Por otro lado, estudios en universitarios han mostrado que existe un mayor consumo de otras sustancias en aquellos que utilizan CE. Por ejemplo, un mayor consumo de alcohol hasta el estado de embriaguez, cigarrillos convencionales, cannabis, entre otros $(4,23)$.
Alteraciones neurológicas debido a la nicotina en adolescentes

La exposición a nicotina durante la adolescencia y la adultez temprana está asociada con un aumento del uso de otras sustancias, que tienen efectos a largo plazo en el desarrollo cerebral (24). El gran uso de este tipo de dispositivos en los adolescentes amenaza los logros de la salud pública, en cuanto al control del consumo de cigarrillo (25).

En la fase de la adolescencia hay una serie de importantes cambios neurobiológicos, pues se da la última poda neuronal. Dado esto, en los adolescentes se ve una disminución en el volumen en la sustancia gris cerebral, que se compensa con un aumento en la sustancia blanca. Esto indica un mayor y mejor proceso de mielinización $(21,26)$. En adolescentes que consumen alcohol se ha encontrado una disminución en este proceso, especialmente en el lóbulo frontal, lo que genera alteraciones en la capacidad de procesamiento y funciones visoespaciales, junto con alteraciones en la motivación (27). Sin embargo, el proceso de mielinización no es homogéneo, por lo que se presentan cambios comportamentales típicos del periodo de la adolescencia. Estos se encuentran dados por la búsqueda de estímulos novedosos y toma de conductas de riesgo, en especial lo referido a conductas sociales $(21,27)$.

También hay una maduración tardía de la inervación de la dopamina, particularmente en la corteza prefrontal anterior. Los niveles máximos de dopamina extracelular se observan durante la adolescencia tardía en el núcleo accumbens, una de las estructuras involucradas en el circuito de recompensa $(21,27,28)$.

Durante la adolescencia, también hay cambios funcionales importantes en el sistema dopaminérgico que produce cambios paralelos en la regulación emocional y la función cognitiva. La estimulación del receptor de dopamina D2 de las interneuronas de rápida aceleración en la corteza prefrontal no aparece hasta la adolescencia tardía con el reclutamiento y la maduración de la actividad local del ácido 
gamma-aminobutírico. Además, durante este tiempo aún se están desarrollando importantes interacciones del receptor D1-NMDA en las neuronas piramidales corticales que son necesarias para el procesamiento cognitivo y de atención del adulto (21).

La activación de los receptores nicotínicos en el circuito de recompensa regula la neurotransmisión dependiente de monoaminas, en especial la dopaminérgica, que está fuertemente ligada con este circuito y al refuerzo de fármacos. La nicotina aumenta la actividad neuronal, medida por la expresión del ARN mensajero, con más fuerza en adolescentes que en adultos en varias regiones relacionadas con la recompensa, incluida la cáscara del núcleo accumbens, la amígdala basolateral y el área ventral tegmental (21).

Las neuronas dopaminérgicas del área tegmental ventral en adolescentes son más sensibles a la potenciación a largo plazo inducida por la nicotina, asociada con la plasticidad de la función del receptor de glutamato.

La exposición inicial a la nicotina induce efectos únicos en la adolescencia en el sistema reticular ascendente (serotoninérgico), llevando a la presencia de síntomas similares a los depresivos en adultos expuestos a nicotina durante la adolescencia, en comparación con los no expuestos, los cuales resuelven al manejo con antidepresivos o a la reexposición de nicotina. Estos cambios están dados por una alteración de la función del receptor de serotonina, con una disminución en la unión del receptor 5-HT2 en las regiones terminales y un cambio en la señalización de los receptores 5-HT1A, desde la estimulación hasta la inhibición de la actividad de la adenilciclasa $(21,29)$.

Sumado a lo anterior, una serie de estudios mostraron el efecto "de entrada al consumo de sustancias psicoactivas" de la nicotina, al encontrar que la exposición prolongada a la nicotina sensibiliza las respuestas de comportamiento de la cocaína y la potenciación a largo plazo en el núcleo accumbens, la amígdala y el hipocampo $(22,30)$. Cabe aclarar que estos efectos son unidireccionales, ya que la cocaína no afecta las respuestas inducidas por la nicotina.
Además, se ha descrito una mayor proporción de trastornos mentales relacionados con la impulsividad y la compulsividad en los usuarios de CE, así como una mayor probabilidad de estrés, depresión o uso de sustancias en individuos con diagnóstico de algún trastorno mental (31). Es importante mencionar que los estudios realizados en aras de evaluar esta relación son todos asociativos y no existen estudios rigurosos que puedan develar causalidad entre estos fenómenos (32). La evidencia más fuerte se ha encontrado por estudios longitudinales que evalúan la asociación entre el uso de CE y el uso de cigarrillo convencional (33).

Aumento en el uso de sistemas electrónicos de administración de nicotina en adolescentes

Desde la publicación, en 1964, acerca de los potenciales daños del cigarrillo, en los adolescentes disminuyó casi cinco veces el tabaquismo, meta contemplada por la American Heart Association. Sin embargo, del 2011 al 2015 aumentó en un 900\% el uso de cigarrillos electrónicos en los adolescentes, y se convirtió así, en el 2014, en el producto de nicotina con mayor uso en esta población. Actualmente, el uso del CE es de dos a tres veces mayor en los adolescentes y adultos jóvenes que en los adultos mayores, lo que implica un retroceso en la disminución del consumo de productos con nicotina en la juventud $(24,34,35)$.

Al revisar su evolución, en el 2013 se estimaba que al menos 263.000 estudiantes de escuela media y superior que nunca habían fumado informaban el consumo actual de CE, y para el 2015, el 16\% de los estudiantes indicaba haber usado CE en los 30 días previos. En la actualidad, al menos un tercio de los usuarios de $\mathrm{CE}$ dijo no haber fumado tabaco antes o no tener tabaquismo activo para el momento de inicio de consumo de CE $(6,36)$. Entre las posibles razones que se encuentran a este fenómeno están el mercadeo y las campañas que muestran a celebridades consumiendo este producto, las imágenes evocativas y la existencia de sabores atractivos $(37,38,39,40)$. Algunos estudios han 
Felipe Botero-Rodríguez, Arturo Marroquín Rivera, Alejandra Leal, et al.

evidenciado una preferencia importante por los dispositivos con sabores tanto en la población adolescentes como en los adultos (41).

Adicionalmente, en Estados Unidos, el 43\% de los estudiantes de último año había "vapeado", lo que representa un $20 \%$ más de los que prueban cigarrillos tradicionales. La mayoría de los adolescentes en escuela media y superior refieren solo usar saborizantes sin nicotina; sin embargo, en el mercado, alrededor del 99\% de los líquidos contienen alguna cantidad de esta sustancia (42).

Por estas razones, los índices de vapeo de nicotina se incrementan cada vez más, pues se han convertido en la sustancia con mayor crecimiento en consumo de los últimos 44 años $(5,43)$. Aumentó 3,4, 8,9 y 10,9 puntos porcentuales en estudiantes de octavo, décimo y duodécimo grados, con prevalencias del 11\%, del 25\% y del 30\%, respectivamente, según el reporte Monitoring the Future, liderado por la Universidad de Michigan (42). Gautier revivió la teoría de la puerta de entrada, exponiendo los $\mathrm{CE}$ como un paso al consumo de otras sustancias, incluido el tabaco convencional (44). En relación con esto, se encontró un aumento en el vapeo de marihuana con una prevalencia anual del $4,4 \%$, del $12,4 \%$ y del $13,1 \%$, respectivamente, en estudiantes de octavo, décimo y duodécimo grados. Se estima que uno de cada tres adolescentes que ha utilizado CE ha "vapeado" marihuana, por medio de aceites con diferentes concentraciones de cannabis. En cuanto al vapeo de sabores sin nicotina, también hubo un aumento con prevalencias del $15 \%$, del $25 \%$ y del $26 \%$, respectivamente $(24,42,45)$.

En Estados Unidos, en la población entre 15 y 17 años, el SEAN con mayor prevalencia de uso fue el JUUL, donde el 9,5\% mencionó haberlo usado; mientras que el 6,1\% refirió un uso actual. De estos individuos, el 55,8\% comentó su uso tres o más días en el último mes. Este consumo se correlacionó con una menor edad, raza blanca, mayor confort financiero, percepción de que es menos nocivo y alta sensación de buscar y consumir tabaco de combustión (15).

En cuanto a la percepción del riesgo por este grupo etario, presenta un bajo puntaje comparado con el uso de otras sustancias. Esta percepción del riesgo, sin embargo, parece aumentar si la sustancia utilizada contiene nicotina, con un riesgo percibido en los de grado octavo del $32 \%$; en los de décimo, del $31 \%$, y en los de duodécimo grado, del 28\% (42).

En nuestra región, el III Estudio Epidemiológico Andino en población universitaria describió una prevalencia de haber consumido alguna vez en su vida estos dispositivos: el 27,4\% en Ecuador, el 16,1\% en Colombia, el 12,8\% en Bolivia y el 12,5\% en Perú, con un mayor consumo en población masculina (46).

Cigarrillo electrónico y salud pública: regulaciones y medidas para contener su crecimiento

El rápido incremento en el consumo y venta de los CE no ha sido proporcional al inicio de regulaciones por parte de los diferentes Estados del mundo. La respuesta ante este creciente mercado ha sido lenta y tiene que ver con las dificultades para regular este producto, al no considerarse estrictamente un producto derivado del tabaco. Adicionalmente, la Organización Mundial de la Salud (OMS) no consideraba nocivos los $\mathrm{CE}$, pues se ha debatido la evidencia existente para declararlos como tales (47). No obstante, para el 2016, 68 países iniciaron la regulación de los $\mathrm{CE}$ en diferentes niveles. De estos 68 países, 22 los regularon utilizando la legislación existente, 25 crearon nuevas políticas, 7 generaron enmiendas a la legislación que existía y 14 emplearon tanto nuevas políticas como enmiendas a las regulaciones previas. Para el 2018 este número aumentó, con 98 países que ya iniciaron la puesta en marcha de leyes nacionales o federales para regular los $\mathrm{CE}$ $(48,49)$. Entre las leyes resaltan: edad mínima de venta, publicidad, promoción y patrocinio, regulaciones en el empaquetado y condiciones producto, reporte y notificación de efectos adversos e implementación de impuestos.

Entre los ejemplos más relevantes de las regulaciones se encuentra el Reino Unido. En un principio se consideró el CE como un 
potencial remplazo del cigarrillo convencional dentro del marco de la cesación del tabaquismo. De esta manera, la regulación en el Reino Unido se hizo a través de la entrada del CE como un medicamento, lo que los limitaba no solo en estándares de productos medicinales, sino también en las condiciones de publicidad. Adicionalmente, en mayo del 2018 se establecieron mínimos de calidad y seguridad de estos productos, implementando nuevas regulaciones para maximizar los beneficios y asegurar la seguridad sobre la base de que los cigarrillos electrónicos son una opción beneficiosa en los consumidores de tabaco (50).

Por otro lado, la respuesta de la Unión Europea fue diferente. Desde el 2014, en la legislación European Union Tobacco Products Directive se incluyó el CE como un dispositivo derivado del tabaco y, por lo mismo, con regulaciones específicas. Entre las más relevantes consideraron una edad mínima de 18 años para su compra, limitaciones en la publicidad, un etiquetado que muestre claramente los ingredientes de los líquidos utilizados, advertencias en los empaques que informen sobre el riesgo de adicción derivado de la nicotina, una concentración máxima de nicotina de todos los dispositivos vendidos en Europa de $20 \mathrm{mg} / \mathrm{ml}$, entre otras (51).

Por su parte, en el 2008, la Food and Drugs Administration (FDA) empezó a confiscar estos dispositivos, y en el 2009, con el surgimiento del Family Smoking Prevention and Tobacco Control Act, documento aprobado, se inició la regulación de estos productos a través de las decisiones de la FDA. Sin embargo, debido a la lenta respuesta de los organismos regulatorios, diferentes estados tomaron precauciones en ausencia de medidas nacionales, como impuestos, políticas libres de humo, precios mínimos establecidos, establecimiento de una edad mínima legal para la compra, limitación de la venta por minoristas y prohibición ciertas clases de productos de tabaco. Finalmente, en el 2016, la FDA logró una ley federal, al incluir de manera definitiva los CE como productos derivados del tabaco y logró: 1) reforzar mecanismos que impidan la adulteración y falsificación de estos productos, 2) presentar el listado de ingredientes y el reporte de constituyentes dañinos o potencialmente dañinos incluidos en el producto, 3) registrar el sitio de fabricación y listado de productos fabricados, 4) controlar la publicidad, 5) prohibir muestras gratis y 6) revisar previamente la comercialización.

Además, se estableció una edad mínima de compra de 18 años (aquellos estados con edades mínimas de compra mayor de 18 años no tienen que cambiarlas), presencia de etiquetado que advierta los potenciales riesgos para la salud y prohibición de su venta en máquinas expendedoras, a menos que estén en un lugar en el que no se acepten menores de edad a toda hora. Cabe aclarar que algunos estados son menos flexibles y establecen edades mínimas superiores $(52,53,54)$.

Otros países han optado por la prohibición de la importación, venta y distribución de los CE en su territorio, como es el caso de Japón, Canadá, México, Brasil, Uruguay, Bahréin, Panamá, entre otros (55).

La preocupación aumenta cuando, además, en esta población se genera un creciente fenómeno de autopromoción en las redes sociales, donde se comparten opiniones favorables, aparte de los conocidos trucos de vapeo, realizados por casi el $80 \%$ de los usuarios de CE, lo cual se relaciona con menor percepción de riesgo y normalización del acto de fumar $(24,34)$. En el 2010, el congreso de Estados Unidos aprobó un documento conocido como Prevent All Cigarette Trafficking Act (PACT Act), cuyo fin es restringir tanto la compra por parte de menores de edad por internet como que fuera gravado el impuesto establecido por la ley. Además, dentro de las regulaciones también está la prohibición del uso de CE en lugares libres de humo de tabaco.

Entre las medidas más efectivas identificadas están los impuestos por la demostrada elasticidad del tabaco, con una relación inversa entre los impuestos y el uso de tabaco. Así, diversos estudios han demostrado que el aumento del precio en un $10 \%$ disminuye el consumo entre un $3-5 \%$ en los adultos y de un $6-7 \%$ en los adolescentes (52). Por su parte, hay poca información sobre el inicio de proyectos educativos dirigidos a la población adolescente 
que busquen informar acerca de los potenciales riesgos del CE. Hasta el 2018, la FDA realizó su primera campaña a gran escala conocida como The Real Cost, aunque estos productos llevan en el mercado estadounidense desde el 2007 (24).

Colombia es parte del Convenio Marco de la OMS para el Control de Tabaco (adoptado mediante la Ley 1109 de 2006), que no considera de manera específica los SEAN. Por esta razón, la OMS ha hecho un llamado para crear una normatividad específica regulatoria hacia estos dispositivos, que no existe en nuestro país. Hasta la fecha se han presentado tres proyectos de ley en el Congreso de la República en relación con este problema. Los dos primeros fueron archivados; mientras que el último busca regular el consumo, la publicidad y la comercialización de los SEAN y similares (56).

Según el reporte Opciones en Colombia para la regulación del uso de los sistemas electrónicos con o sin dispensación de nicotina y similares: un resumen de evidencias para política (56) y la Declaración de sociedades científicas colombianas acerca del uso de sistemas (cigarrillos) electrónicos de administración de nicotina (SEAN) y sistemas similares sin nicotina (SSSN), la opción más apropiada para nuestro contexto parece ser la misma que se ha usado más frecuentemente en el mundo, que busca tratar los SEAN como productos sucedáneos del tabaco, para poder ser acogido por la Ley 1335 de 2009, cuyo objetivo es garantizar el derecho a la salud de la población, protegiéndolos de los efectos del consumo de tabaco y sus derivados, así como la exposición al humo de cigarrillo, especialmente a la población menor de 18 años.

Así mismo, los documentos anteriores señalan la importancia de no incluir estos dispositivos como tratamiento para el cese del consumo del cigarrillo, dada su pobre evidencia frente a esta patología; aparte de que alientan modificaciones de la ley mencionada para la restricción al acceso de estos dispositivos. Además, proponen realizar campañas de psicoeducación y prevención primaria, en las que se informe mejor el efecto nocivo que estos dispositivos traen a la salud general (57).

\section{Conclusión}

El efecto nocivo de la nicotina en el neurodesarrollo de los adolescentes y, por lo tanto, en general de los CE, así como su creciente consumo mundial, es un llamado urgente a la instauración de medidas concordantes. Esto sugiere un riesgo elevado para la salud mental de los adolescentes, que, sumado a una pobre regulación en nuestro país, genera la necesidad de fortalecer los sistemas de prevención e intervención para el consumo de nicotina en sus diferentes modalidades.

\section{Conflicto de interés}

Los autores declaran no presentar ningún conflicto de interés.

\section{Referencias}

1. Grana R, Benowitz N, Glantz SA. E-Cigarettes a scientific review. Circulation. 2014;129:1972-86.

2. Monraz-Pérez S, Regalado-Pineda J, Pérez-Padilla R. El cigarrillo electrónico: Peligro u oportunidad. Neumol Cir Torax. 2015;74(2):82-6.

3. Orellana-Barrios MA, Payne D, Mulkey Z, Nugent K. Electronic cigarettes-a narrative review for clinicians. Am J Med. 2015 Jul;128(7):674-81.

4. Biyani S, Derkay CS. E-cigarettes: An update on considerations for the otolaryngologist. Int J Pediatr Otorhinolaryngol. 2017 Mar;94(2017):14-6.

5. Jenssen BP, Wilson KM. Tobacco control and treatment for the pediatric clinician: practice, policy, and research updates. Acad Pediatr. 2017 Apr;17(3):233-42.

6. Dinakar C, O'Connor GT. The Health effects of electronic 
cigarettes. N Engl J Med. 2016 Oct 6;375(14):1372-81.

7. Walley SC, Wilson KM, Winickoff JP, Groner J. A public health crisis: electronic cigarettes, vape, and JUUL. Pediatrics. 2019 Jun;143(6). https://doi .org/10.1542/peds.2018-2741

8. St. Helen G, Eaton DL. Public health consequences of e-cigarette Use. JAMA Intern Med. 2018 Jul;178(7):984.

9. Litt MD, Duffy V, Oncken C. Cigarette smoking and electronic cigarette vaping patterns as a function of e-cigarette flavourings. Tob Control [Internet]. $2016 \mathrm{Nov}$ [citado $2019 \mathrm{Mar}$ 19];25(Suppl 2):ii67-72. Disponible en: http://tobaccocontrol.bmj.com/loo kup/doi/10.1136/tobaccocontrol-2016 $-053223$

10. Breland A, Soule E, Lopez A, Ramoa C, El-Hellani A, Eissenberg T. Electronic cigarettes: what are they and what do they do? Ann N Y Acad Sci. 2017 Apr;1394(1):5-30.

11. Etter J-F, Bullen C, Flouris $\mathrm{AD}$, Laugesen $\mathrm{M}$, Eissenberg $\mathrm{T}$. Electronic nicotine delivery systems: a research agenda. Tob Control [Internet]. 2011 May 1 [citado 2019 May 23];20(3):243-8. Disponible en: http://www.ncbi.nlm.nih.gov/pubm ed/21415064

12. Zulkifli A, Abidin EZ, Abidin NZ, Amer Nordin AS, Praveena SM, Syed Ismail SN, et al. Electronic cigarettes: a systematic review of available studies on health risk assessment. Rev Environ Health. 2018 Mar;33(1):43-52.

13. Harrell PT, Simmons VN, Correa JB, Padhya TA, Brandon $\mathrm{TH}$. Electronic nicotine delivery systems ("e-cigarettes"): review of safety and smoking cessation efficacy. Otolaryngol Head Neck Surg. 2014 Sep;151(3):381-93.
14. Mitchko J, Lewis S, Marynak KL, Shannon C, Burnette D, King BA. E-cigarettes and young people: communicating an emerging public health risk. Am J Heal Promot. 2019 Mar 14;089011711983551.

15. Vallone DM, Bennett M, Xiao H, Pitzer L, Hair EC. Prevalence and correlates of JUUL use among a national sample of youth and young adults. Tob Control. 2018 Oct 29; tobaccocontrol-2018-054693.

16. Marsot A, Simon N. Nicotine and cotinine levels with electronic cigarette: a review. Int J Toxicol. 2016;35(2):179-85.

17. Fearon IM, Eldridge AC, Gale N, Mcewan M, Stiles MF, Round EK. Nicotine pharmacokinetics of electronic cigarettes: A review of the literature. 2018;

18. Fadus MC, Smith TT, Squeglia LM. The rise of e-cigarettes, pod mod devices, and JUUL among youth: Factors influencing use, health implications, and downstream effects. Drug Alcohol Depend. 2019 May;201:85-93.

19. Unger M, Unger DW. E-cigarettes/ electronic nicotine delivery systems: a word of caution on health and new product development. J Thorac Dis. 2018 Aug;10(Suppl 22):S2588-92.

20. Aicher BO, Frishman WH. Electronic cigarettes: questions in the mist. Cardiol Rev. 2016;24(6):261-7.

21. Yuan M, Cross SJ, Loughlin

SE, Leslie FM. Nicotine and the adolescent brain. J Physiol [Internet]. 2015 Aug 15 [citado 2019 May 23];593(16):3397-412. Disponible en: http://www.ncbi.nlm.ni h.gov/pubmed/26018031

22. England LJ, Bunnell RE, Pechacek TF, Tong VT, McAfee TA. Nicotine and the developing human: a neglected 
element in the electronic cigarette debate. Am J Prev Med. 2015 Aug;49(2):286-93.

23. Giroud C, de Cesare M, Berthet A, Varlet V, Concha-Lozano N, Favrat B. E-Cigarettes: A review of new trends in cannabis use. Int J Environ Res Public Health. 2015 Aug;12(8):9988-10008.

24. Chadi N, Hadland SE, Harris SK. Understanding the implications of the "vaping epidemic" among adolescents and young adults: A call for action. Subst Abus. 2019 Mar;1-4.

25. Jenssen BP, Boykan R. Electronic cigarettes and youth in the United States: A call to action (at the local, national and global levels). Child (Basel, Switzerland). 2019 Feb;6(2).

26. Gogliettino AR, Potenza MN, Yip SW. White matter development and tobacco smoking in young adults: A systematic review with recommendations for future research. Drug Alcohol Depend [Internet]. 2016 May [citado 2019 May 2];162:26-33. Disponible en: https://inkinghub.elsevier.com/ret rieve/pii/S0376871616000909

27. Koob GF, Volkow ND. Neurobiology of addiction: a neurocircuitry analysis. The Lancet Psychiatry [Internet]. 2016 Aug [citado 2019 May 23];3(8):760-73. Disponible en: http://www.ncbi.nlm.ni h.gov/pubmed/27475769

28. Philpot RM, Wecker L, Kirstein CL. Repeated ethanol exposure during adolescence alters the developmental trajectory of dopaminergic output from the nucleus accumbens septi. Int J Dev Neurosci [Internet]. 2009 Dec [citado 2019 May 23];27(8):805-15. Disponible en: http://www.ncbi.nlm.ni h.gov/pubmed/19712739

29. Iñiguez $\mathrm{SD}$, Warren $\mathrm{BL}$, Parise EM, Alcantara LF,
Schuh B, Maffeo ML, et al. Nicotine exposure during adolescence induces a depression-like state in adulthood. Neuropsychopharmacology [Internet]. 2009 May [citado 2019 May 23];34(6):1609-24. Disponible en: http://www.ncbi.nlm.nih.gov/pubm ed/19092782

30. Levine A, Huang Y, Drisaldi B, Griffin EA, Pollak DD, Xu S, et al. Molecular mechanism for a gateway drug: epigenetic changes initiated by nicotine prime gene expression by cocaine. Sci Transl Med [Internet]. 2011 Nov 2 [citado 2019 May 23];3(107):107ra109-107ra109. Disponible en: http://www.ncbi.nlm.ni h.gov/pubmed/22049069

31. Grant JE, Lust K, Fridberg DJ, King AC, Chamberlain SR. E-cigarette use (vaping) is associated with illicit drug use, mental health problems, and impulsivity in university students. Ann Clin Psychiatry. 2019;31(1):27-35.

32. Perikleous EP, Steiropoulos P, Paraskakis E, Constantinidis TC, Nena E. E-Cigarette Use Among Adolescents: An overview of the literature and future perspectives. Front Public Heal. 2018;6:86.

33. Soneji S, Barrington-Trimis JL, Wills TA, Leventhal AM, Unger JB, Gibson LA, et al. Association between initial use of ecigarettes and subsequent cigarette smoking among adolescents and young adults: a systematic review and meta-analysis. JAMA Pediatr. 2017 Aug;171(8):788-97.

34. Bhatnagar A, Whitsel LP, Ribisl KM, Bullen C, Chaloupka F, Piano $\mathrm{MR}$, et al. Electronic cigarettes: a policy statement from the American Heart Association. Circulation. 2014 Oct;130(16):1418-36.

35. Carroll Chapman SL, Wu L-T. Ecigarette prevalence and correlates of 
use among adolescents versus adults: a review and comparison. J Psychiatr Res. 2014 Jul;54:43-54.

36. Wang M, Wang J-W, Cao S-S, Wang H-Q, Hu R-Y. Cigarette smoking and electronic cigarettes use: a metaanalysis. Int J Environ Res Public Health. 2016 Jan;13(1).

37. Payne JD, Orellana-Barrios M, Medrano-Juarez R, Buscemi D, Nugent $\mathrm{K}$. Electronic cigarettes in the media. Proc (Bayl Univ Med Cent). 2016 Jul;29(3):280-3.

38. Hoffman AC, Salgado RV, Dresler C, Faller RW, Bartlett C. Flavour preferences in youth versus adults: a review. Tob Control. 2016 Nov;25(Suppl 2):ii32-9.

39. Smith L, Brar K, Srinivasan K, Enja M, Lippmann S. E-cigarettes: How "safe" are they? J Fam Pract. 2016 Jun;65(6):380-5.

40. Durmowicz EL. The impact of electronic cigarettes on the paediatric population. Tob Control. 2014 May;23 Suppl 2:ii41-6.

41. Zare S, Nemati M, Zheng Y. A systematic review of consumer preference for e-cigarette attributes: Flavor, nicotine strength, and type. PLoS One. 2018;13(3):e0194145.

42. Johnston LD, Miech RA, O'malley PM, Bachman JG, Schulenberg JE, Patrick ME. Monitoring the future national survey results on drug use 1975-2017: Overview [Internet]. Michigan; 2018 [citado 2019 May 23]. Disponible en: http://www.monitoringt hefuture.org/pubs/monographs/mtf-ov erview2017.pdf

43. Greenhill R, Dawkins L, Notley C, Finn MD, Turner JJD. Adolescent awareness and use of electronic cigarettes: a review of emerging trends and findings. J Adolesc Health. 2016 Dec;59(6):612-9.
44. Gautier S, Kinouani S, Raherison C. Vapoter favorise-t-il le tabagisme chez les adolescents et les jeunes adultes\#? Revue de la littérature. Sante Publique (Paris). 2017;29(3):333-40.

45. Breitbarth AK, Morgan J, Jones AL. E-cigarettes-An unintended illicit drug delivery system. Drug Alcohol Depend. 2018 Nov;192:98-111.

46. Oficina de las Naciones Unidas Contra la Droga y el Delito. III Estudio Epidemiológico Andino sobre Consumo de Drogas en la Población Universitaria, Informe Regional, 2016 [Internet]. Lima; 2017 [citado 2019 Jul 16]. Disponible en: https://www.unodc .org/documents/colombia/2017/Octub re/Informe_Universitario_Regional.pd $\mathrm{f}$

47. Jankowski M, Brozek G, Lawson J, Skoczynski S, Zejda JE. E-smoking: Emerging public health problem? Int J Occup Med Environ Health. 2017 May;30(3):329-44.

48. World Bank Group. E-cigarettes: use and taxation wbg global tobacco control program team working note [Internet]. Washington; 2019 [citado 2019 May 23]. Disponible en: http://documents.worldbank.org/c urated/en/356561555100066200/pdf/E -Cigarettes-Use-and-Taxation.pdf

49. Rose A, Filion KB, Eisenberg MJ, Franck C. Electronic cigarettes: A comparison of national regulatory approaches. Can J Public Health. 2015 Oct;106(6):e450-3.

50. Public Health England. Ecigarettes: an evidence update $\mathrm{A}$ report commissioned by Public Health England. Londres; 2015.

51. Unión Europea. Directiva 2014/40/ UE del Parlamento Europeo y del Consejo de 3 de abril de 2014 [Internet]. 2014 [citado 2019 Jun 10]. Disponible en: https://ec.europa.eu/he 
alth/sites/health/files/tobacco/docs/dir _201440_es.pdf

52. Atwater P, Fradkin N, Medeiros E, Gilroy JH, Halperin A. E-cigarettes and public health: policy options for washington state. Washington; 2015.

53. Barraza LF, Weidenaar KE, Cook LT, Logue AR, Halpern MT. Regulations and policies regarding e-cigarettes. Cancer. 2017 Aug 15;123(16):3007-14.

54. Drope JJ, Cahn Z, Kennedy R, Liber AC, Stoklosa M, Henson R, et al. Key issues surrounding the health impacts of electronic nicotine delivery systems (ENDS) and other sources of nicotine. CA Cancer J Clin. 2017 Nov;67(6):449-71.

55. Instituto Nacional de Vigilancia de Alimentos y Medicamentos. Reglamentación cigarrillos electrónicos: consideraciones generales basadas en la evidencia [Internet]. Bogotá; 2016. Disponible en: https://paginaweb.invima.gov.co/i mages/pdf/anuncios/REVISION-CIG ARRILLO-ELECTRONICO_EHOC_ 10_10_2016_REVI.pdf

56. Pulido Álvarez A, Pinzón Silva DC, Rodríguez NI, Sandoval Salinas C, Pinzón Flórez CE, Díaz Ortega MH, Mejía A, Santacruz JC, Calderón Herrera J. Opciones en Colombia para la regulación del uso de los sistemas electrónicos con o sin dispensación de nicotina y similares: un resumen de evidencias para política (policy brief) [Internet]. Bogotá: Instituto de Evaluación Tecnológica en Salud; 2018 [citado 2019 Jul 16]. Disponible en: http://www.iets.org.co/Archivos/3/ Policy_brief_version_completa.pdf

57. Sociedad Colombiana de Medicina Familiar, Asociación Colombiana de Neumología y Cirugía de Tórax, Asociación Colombiana de Neumología Pediátrica, Asociación
Colombiana de Medicina Interna, Sociedad Colombiana de Cardiología y Cirugía Cardiovascular, Asociación Colombiana de Hematología y Oncología, Asociación Colombiana de Patología. Declaración de sociedades científicas colombianas acerca del uso de sistemas (cigarrilllos) electrónicos de administración de nicotina (SEAN) y sistemas similares sin nicotina (SSSN) [Internet]. Bogotá D.C.; 2018. Disponible en: http://socmef.com/socm ef/images/pdf/posicion_sociedades_cie ntificas_sean.pdf 